\title{
Rydberg-Dressed Magneto-optical Trap
}

\author{
A. D. Bounds, N. C. Jackson, R. K. Hanley, R. Faoro, E. M. Bridge, P. Huillery, and M. P. A. Jones" \\ Joint Quantum Centre Durham-Newcastle, Department of Physics, Durham University, Durham DH1 3LE, United Kingdom
}

(Received 12 January 2018; revised manuscript received 5 March 2018; published 4 May 2018)

\begin{abstract}
We propose and demonstrate the laser cooling and trapping of Rydberg-dressed Sr atoms. By offresonantly coupling the excited state of a narrow $(7 \mathrm{kHz})$ cooling transition to a high-lying Rydberg state, we transfer Rydberg properties such as enhanced electric polarizability to a stable magneto-optical trap operating at $<1 \mu \mathrm{K}$. Simulations show that it is possible to reach a regime where the long-range interaction between Rydberg-dressed atoms becomes comparable to the kinetic energy, opening a route to combining laser cooling with tunable long-range interactions.
\end{abstract}

DOI: 10.1103/PhysRevLett.120.183401

The strong interactions between Rydberg atoms have led to numerous experimental breakthroughs in many-body quantum simulation [1-4], quantum information processing [5-7], and quantum optics [8-12]. To take advantage of coherent dynamics, these realizations have focused on timescales shorter than the lifetime of the Rydberg state. However, there is growing interest in extending the investigation time of Rydberg ensembles for applications in quantum simulation and metrology [13-15]. A promising method is to off-resonantly couple the ground state to a Rydberg state, resulting in the controlled admixture of some interacting Rydberg character [16-20]. This Rydberg dressing approach could enable the realization of supersolids [21-24], frustrated or topological quantum magnetism [25-28], or spin squeezing for metrology [15,17]. Experimentally, Rydberg dressing has been demonstrated for two atoms [29] and in optical lattices [3,30], but it seems to be more challenging in randomly distributed ensembles due to uncontrolled loss mechanisms [31-34].

In this Letter we introduce a new scheme where Rydberg dressing is applied to an excited state undergoing spontaneous emission (Fig. 1). We show that Rydberg-dressed atoms can be laser cooled to sub-microkelvin temperatures and trapped in a magneto-optic trap (MOT), while simultaneously acquiring properties of the Rydberg state such as enhanced sensitivity to dc electric fields. The result is a hybrid magneto-electro-optical trap controllable by electric as well as magnetic fields. We show that the Rydbergdressed MOT can operate in a regime where the interaction strength is comparable to the dissipation and the kinetic energy, and with a lifetime that exceeds that of the Rydberg

Published by the American Physical Society under the terms of the Creative Commons Attribution 4.0 International license. Further distribution of this work must maintain attribution to the author(s) and the published article's title, journal citation, and DOI. state by a factor of $\sim 70$. Although laser cooling of Rydberg-dressed atoms has been proposed to protect crystalline phases against dissipative effects [20], active cooling of Rydberg gases is a relatively unexplored area $[35,36]$ where interesting physics could arise from the presence of cooling and the mechanical effect of the interactions. Examples could include Sisyphus-like cooling $[37,38]$ induced by the Rydberg-dressed potential or, in the spirit of antiblockade experiments [39], cooperative cooling where the collective scattering of multiple photons by groups of atoms dominates over single particle cooling.

The laser cooling and Rydberg dressing scheme is shown in Fig. 1(a). The goal is to dress the upper state $|e\rangle$ of the
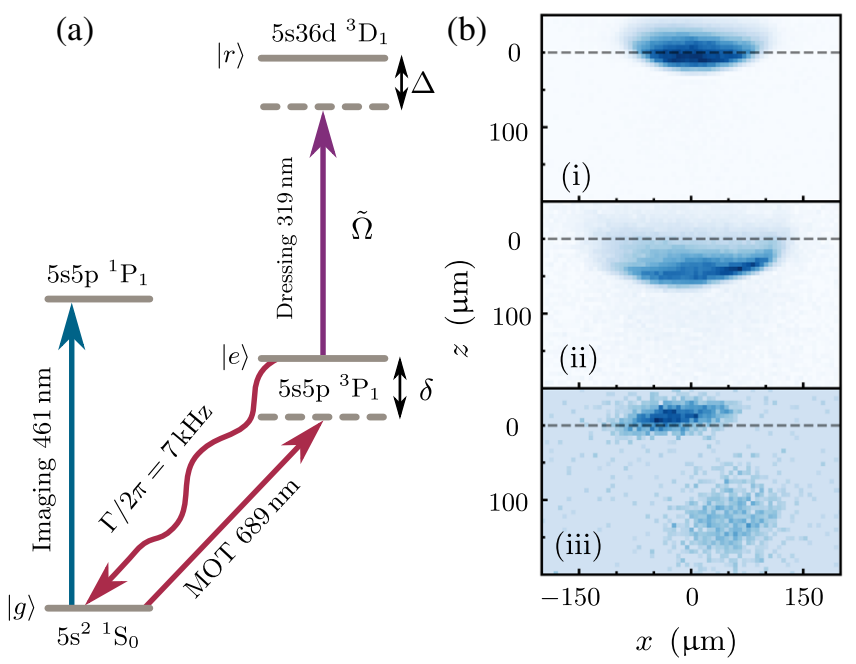

FIG. 1. (a) A MOT operating on the $689 \mathrm{~nm}$ intercombination transition is dressed by off-resonantly coupling state $|e\rangle$ to a Rydberg state $|r\rangle$. The strong $461 \mathrm{~nm}$ transition is used for imaging. (b) Images (i) of the undressed MOT with $\delta / 2 \pi=-110 \mathrm{kHz}$, (ii) after $5 \mathrm{~ms}$ dressing with $\delta / 2 \pi=-110 \mathrm{kHz}$, and (iii) after $5 \mathrm{~ms}$ dressing with $\delta / 2 \pi=+190 \mathrm{kHz}$. Here, $\tilde{\Omega} / 2 \pi=4 \mathrm{MHz}$ and $\Delta / 2 \pi=12 \mathrm{MHz}$. The dashed line indicates the position of the undressed MOT. 
cooling transition by coupling it to a Rydberg state $|r\rangle$ with Rabi frequency $\tilde{\Omega}$ and detuning $\Delta$. In the weak dressing limit $\tilde{\Omega} \ll \Delta$, this creates a new Rydberg-dressed excited state $|\tilde{e}\rangle \approx|e\rangle-\epsilon|r\rangle$ with dressing fraction $\epsilon=\tilde{\Omega} /(2 \Delta)$ that experiences an ac Stark shift $\delta_{\text {ac }}=\Delta\left(\sqrt{1+4 \epsilon^{2}}-1\right) / 2$. Unlike previous work [31], the detuning $\delta$ of the cooling laser is small, such that a significant population is transferred to state $|\tilde{e}\rangle$.

In addition to the ac Stark shift, two atoms in the dressed state $|\tilde{e}\rangle$ separated by a distance $r$ experience a dressed interaction potential $[18,21,22] V(r)=V_{0}\left[1+\left(r / R_{c}\right)^{6}\right]^{-1}$ with a peak magnitude $V_{0}=\hbar \tilde{\Omega}^{4} / 8|\Delta|^{3}$ and a length scale $R_{c}=\left|C_{6} / 2 \hbar \Delta\right|^{1 / 6}$. Here, $C_{6}$ is the van der Waals coefficient associated with the Rydberg state $|r\rangle$. In our experiment, $R_{c}$ has typical values of $1 \rightarrow 5 \mu \mathrm{m}$. For reasonable choices of $\tilde{\Omega}$ and $\Delta, \delta_{\mathrm{ac}}$ and $V_{0}$ typically range from 0 to $100 \mathrm{kHz}$, far smaller than the linewidth of most cooling transitions.

We circumvent this limitation by using the $5 s^{21} S_{0} \rightarrow$ $5 s 5 p^{3} P_{1}$ intercombination line in ${ }^{88} \mathrm{Sr}$ with linewidth $\Gamma / 2 \pi=7 \mathrm{kHz}$. Thus, $V_{0}$ is comparable to both the linewidth and the kinetic energy $V_{0} \approx\left\{\hbar \Gamma, k_{B} T\right\}$, while the high MOT density $\rho \approx 10^{12} \mathrm{~cm}^{-3}$ means that $R_{c}$ can exceed the spacing between dressed-state atoms. In this regime, dressed atoms within a range $r<R_{c}$ experience a collective energy shift $V_{c}=N_{c}\left(N_{c}-1\right) V_{0} / 2$ [18,21], where $N_{c}=\eta \rho 4 \pi R_{c}^{3} / 3$. This collective enhancement for $N_{c} \gg 1$ enables the strongly interacting regime $\left(V_{c} / N_{c} \geq \hbar \Gamma, k_{B} T\right)$ to be reached even though the average fraction of atoms in the excited state $\eta<0.1$ is small (see the Supplemental Material [40]).

The experiments begin with the formation of a MOT on the intercombination line; the loading procedure and apparatus are described in Refs. [49,50]. The MOT was operated with an intensity per beam of $I=4 I_{\text {sat }}-10 I_{\text {sat }}$, where $I_{\text {sat }}$ is the saturation intensity, resulting in a $1 / e^{2}$ cloud radii of $30 \mu \mathrm{m}[100 \mu \mathrm{m}$ ] in the vertical $(z)$ [horizontal (x)] direction, and the temperature $T_{z}=800 \mathrm{nK}$. The trap forms where the detuning $\delta$ of the cooling laser matches the Zeeman shift from the quadrupole magnetic field and the gravitational sag [51]. As a result, the detuning seen by the atoms is largely fixed and instead $\delta$ determines the cloud shape and position with a sensitivity of $\sim 10 \mathrm{kHz}$. To form a Rydberg-dressed MOT, the excited state $|e\rangle=$ $5 s 5 p^{3} P_{1}$ was coupled to the Rydberg state $|r\rangle=$ $5 s 36 d^{3} D_{1}$, for which the interactions are weakly attractive [52]. The horizontally propagating dressing laser produced up to $250 \mathrm{~mW}$ at $319 \mathrm{~nm}$ [53], and it was linearly polarized in the vertical $(z)$ direction. The $1 / e^{2}$ beam radius was $120 \mu \mathrm{m}(160 \mu \mathrm{m})$ in the horizontal (vertical) direction, and the Rabi frequency $\tilde{\Omega}$ was measured using Autler-Townes splitting. After dressing for a time $t_{d}$, the cloud was imaged at an angle of $30^{\circ}$ to the coupling beam via absorption on the $461 \mathrm{~nm}$ transition.
The effect of the dressing laser is shown in Fig. 1(b). For the chosen parameters $\epsilon^{2}=0.03$ and $N_{c}=0.5$, such that the MOT is effectively in the noninteracting regime. Figures 1(b)(i) and 1(b)(ii) show that the primary consequence of the dressing is a significant vertical shift of the MOT since the ac Stark shift $\delta_{\mathrm{ac}}$ adds to the detuning $\delta$ experienced by the atoms. By simultaneously adjusting the detuning such that $\delta \rightarrow \delta-\delta_{\mathrm{ac}}$ during the dressing stage, we compensate for this ac Stark shift, and the MOT remains at its original position [Fig. 1(b)(iii)]. The vertical shift is eliminated for a detuning compensation of $+300 \mathrm{kHz}$, close to the calculated peak ac Stark shift of $+325 \mathrm{kHz}$. Importantly, the cooling laser is now blue detuned with respect to the bare transition. Therefore, the compensated MOT traps only Rydberg-dressed atoms; undressed atoms outside the dressing beam are observed falling away [Fig. 1(b)(iii)].

To verify that the Rydberg atoms are laser cooled as well as trapped, we measured the temperature (using ballistic expansion) as a function of $t_{d}$, as shown in Fig. 2. Heating is observed in the first millisecond, but the temperature subsequently returns to that of the initial undressed MOT, showing that laser cooling is active during the dressing. To gain further insight, we adapted the quantitative Monte Carlo simulations described in Ref. [54], adding the spatially dependent ac Stark shift due to the dressing beam (see the Supplemental Material [40]). The simulation is in quantitative agreement with the data, with the only fit parameter being the position of the dressing beam relative to the center of the quadrupole magnetic field, in this case $-20 \mu \mathrm{m}$ below and $60 \mu \mathrm{m}$ to the side of the quadrupole center. These simulations showed that the initial heating arises because the spatial dependence of the ac Stark shift transiently leads to increased scattering, as atoms find themselves at the "wrong" detuning. We confirmed experimentally that the heating depends on the UV beam alignment, reflecting the sensitivity of the MOT to small changes in detuning. Cooling then occurs as the

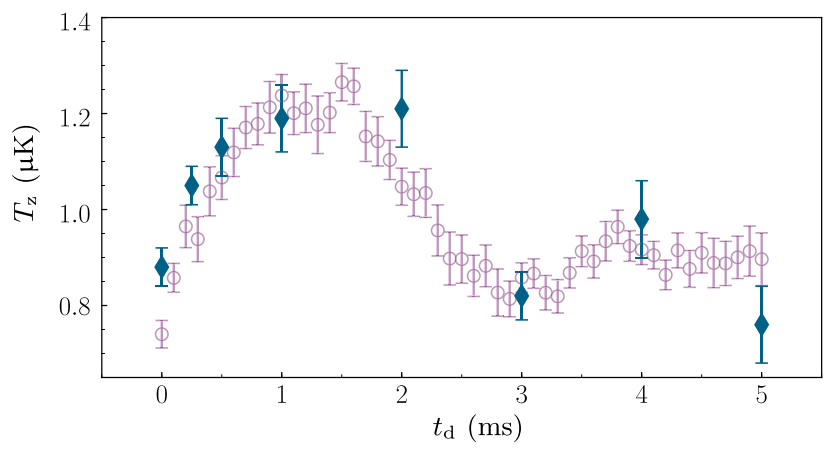

FIG. 2. Measured (blue diamonds) and simulated (purple circles) temperature in the vertical $(z)$ direction $T_{z}$ versus $t_{d}$ for $\tilde{\Omega} / 2 \pi=4 \mathrm{MHz}, \Delta / 2 \pi=12 \mathrm{MHz}$ and $\delta / 2 \pi=190 \mathrm{kHz}$. Error bars indicate the standard error of the mean. 
cloud shape adapts to match the new spatially dependent resonance condition.

An essential aspect of Rydberg dressing is that the dressed atoms acquire characteristics of the Rydberg state. To show that this occurs alongside cooling and trapping, we demonstrate that the dressed MOT becomes sensitive to dc electric fields due to the high polarizability $\alpha_{r}$ of the Rydberg state. A static electric field was applied in the horizontal $(x, y)$ plane at an angle of $30^{\circ}$ to the coupling beam during the dressing time $t_{d}=10 \mathrm{~ms}$ using segmented ring electrodes [50]. As shown in Fig. 3(a), the electric field has a drastic effect on the shape, density, and position of the MOT. At low field strength [Fig. 3(a)(i)], the dc Stark shift of the Rydberg state $\Delta_{\mathrm{dc}}$ is small [Fig. 3(b)], such that $\Delta>\left\{\tilde{\Omega}, \Delta_{\mathrm{dc}}\right\}$ and the dressed state picture remains valid. In this case, atoms in $|\tilde{e}\rangle$ acquire a polarizability given by $\alpha_{\mathrm{e}}=\epsilon^{2} \alpha_{r}$. For the parameters of Fig. 3, $\alpha_{\mathrm{e}} \approx 10^{-33} \mathrm{C} \mathrm{m}^{2} \mathrm{~V}^{-1}$, which is $\sim 10^{5}$ times larger than that of the bare $5 s 5 p^{3} P_{J}$ states $[55,56]$. The resulting change in $\delta$ leads to an associated position shift. For larger fields, $\widetilde{\Omega} \approx \Delta-\Delta_{\mathrm{dc}}$ and the variation in ac Stark shift is associated with population transfer to $|r\rangle$, leading to loss [Fig. 3(a)(ii)].

As the MOT forms below the center of the magnetic quadrupole due to gravity, the atoms primarily experience a vertical magnetic and horizontal electric field. Together with the laser polarization, this results in stronger coupling to the $36 d\left|m_{J}\right|=0$ state than the $36 d\left|m_{J}\right|=1$ state [57].

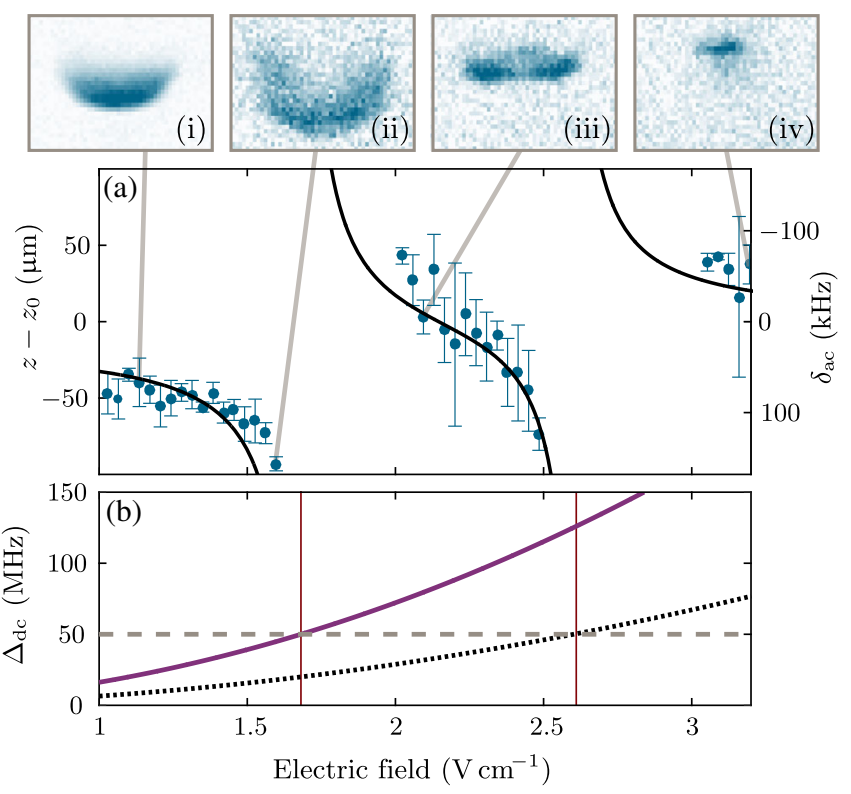

FIG. 3. (a) Measured (blue circles) and predicted (black line) changes in the vertical MOT position versus the electric field with $t_{d}=10 \mathrm{~ms}, \tilde{\Omega} / 2 \pi=4 \mathrm{MHz}$, and $\delta / 2 \pi=-110 \mathrm{kHz}$. Images (i)(iv) correspond to $1.1,1.6,2.1$, and $3.2 \mathrm{~V} \mathrm{~cm}^{-1}$, respectively. (b) dc Stark shift of the $\left|m_{J}\right|=1$ (dotted black line) and $\left|m_{J}\right|=0$ (purple line) components of the $36 d^{3} D_{1}$ state. The dashed grey line indicates $\Delta / 2 \pi=50 \mathrm{MHz}$. The vertical lines (red) denote resonance positions.
However, the slight variation of the magnetic field direction across the cloud means that atoms in the center and wings experience different coupling strengths, causing the cloud to distort. This effect is strongest for values of the electric field for which $\Delta$ lies between the resonances associated with the $\left|m_{J}\right|=0,1$ components [Fig. 3(a)(iii)]. At higher field strengths, the sign of the ac Stark shift is reversed [Fig. 3(a)(iv)], and the MOT forms above its initial position. Away from resonances, the shift in the position of the cloud is in good agreement with that predicted from the ac Stark shift [Fig. 3(a)], provided that the stronger coupling to the $36 d\left|m_{J}\right|=0$ state is taken into account. Interestingly, a trap exists for all values of the electric field for which the dressing beam is sufficiently far from resonance. The Rydberg-dressed MOT can thus be viewed as a novel hybrid trap whose size, shape, and position can be controlled by a relatively weak electric as well as magnetic field. This enhanced sensitivity to spatially dependent electric fields could find applications in electrometry for optical lattice clocks [58].

The observation of interactions requires sufficient interaction strength $\bar{V}_{c}=V_{c} /\left(N_{c} \hbar \Gamma\right) \geq 1$ to be maintained over the $\sim 3 \mathrm{~ms}$ timescale associated with the atomic motion (Fig. 2). Measurements of the atom number as a function of $t_{d}$ are shown in Fig. 4(a). For the $5 s 36 d^{3} D_{1}$ state considered so far, we observe rapid loss with a decay constant of $0.4 \pm 0.1 \mathrm{~ms}$ at short times. Using a microchannel plate detector, we determined that this loss is associated with ionization [57]. The dc Stark shift due to ions in the cloud brings the dressing laser into resonance, leading to enhanced Rydberg excitation and loss. Although we observed that this effect can be partially suppressed by applying a dc electric field, a better approach is to switch to a Rydberg state with the opposite sign of dc Stark shift. The $5 s n d^{3} D_{2}$ states (for $n<37$ ) have repulsive van der Waals interactions and a positive Stark shift, such that ions shift nearby atoms out of resonance with the red-detuned dressing laser. For the $n=36,37$ states, we clearly observe reduced trap loss at short times with an electric fieldindependent lifetime of $1.2 \pm 0.5 \mathrm{~ms}$ for $n=36$, which is $\sim 70$ times larger than the measured lifetime of the Rydberg state $(\tau=18 \pm 1 \mu \mathrm{s})$. A drawback of working with the $5 s n d^{3} D_{2}$ states is that the change in sign of $\delta_{\text {ac }}$ combined with the Gaussian spatial profile of the dressing beam leads to weaker confinement (see the Supplemental Material [40]), limiting the maximum interaction strength we could achieve to $\bar{V}_{c}=0.15(n=36)$. This weak confinement also appears to cause the residual density-dependent decay at short times. At $t_{d}=3 \mathrm{~ms}, 20 \%$ of the atoms remain, and the subsequent decay (lifetime $5 \pm 1 \mathrm{~ms}$ ) is compatible with the decay of the undressed MOT after the UV beam is switched off [Fig. 4(a)].

Surprisingly, the density [Fig. 4(b)] decays faster than the atom number. Images of the MOT [the insets of Fig. 4(b)] show that this is due to a significant horizontal 


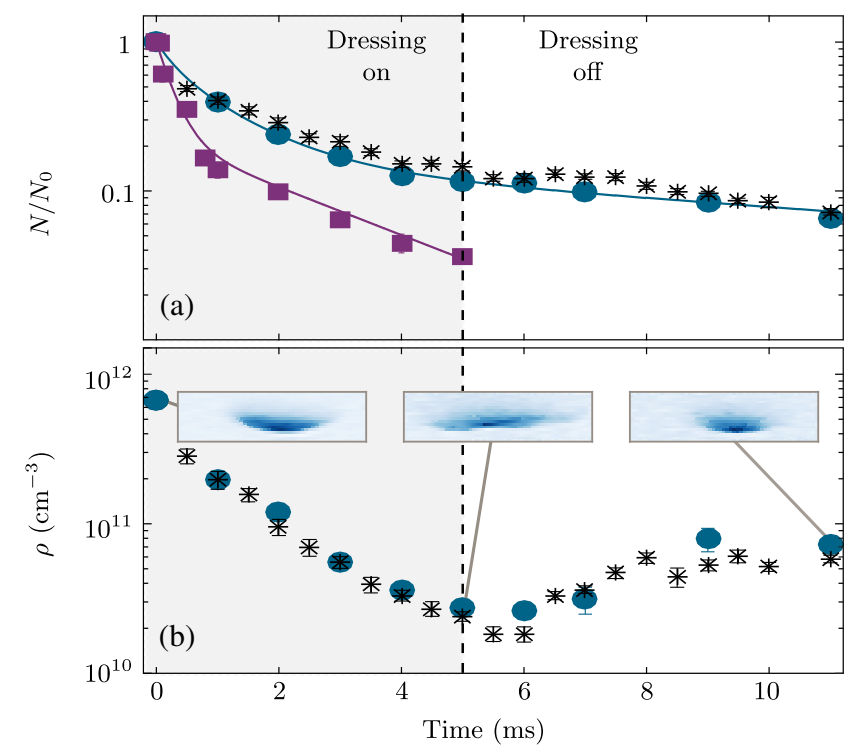

FIG. 4. (a) Normalized atom number $N / N_{t=0}$ as a function of time for the $5 s 36 d^{3} D_{1}$ (purple squares), $5 s 36 d^{3} D_{2}$ (blue circles), and $5 s 37 d^{3} D_{2}$ (black stars) Rydberg states. The solid lines represent double exponential fits. Shading indicates where the dressing laser is on (b) density, $\rho$, versus time for the $5 s 36 d^{3} D_{2}$ (blue circles) and $5 s 37 d^{3} D_{2}$ (black stars) states. Here, $\tilde{\Omega} / 2 \pi=$ $4 \mathrm{MHz}, \Delta / 2 \pi=12 \mathrm{MHz}\left(5 s 36 d^{3} D_{1}\right)$ and $\tilde{\Omega} / 2 \pi=5 \mathrm{MHz}$, $\Delta / 2 \pi=30 \mathrm{MHz}\left[5 s(36,37) d^{3} D_{2}\right]$. (Insets) Images at indicated times for the $5 s 36 d^{3} D_{2}$ state.

expansion of the cloud during dressing. After the dressing laser is switched off, the cloud returns to its original size and the density is largely restored. This expansion is independent of the principal quantum number over the range $n=34-37$, where we expect $N_{c}$ to vary from 0.7 to 25 [59] and $\bar{V}_{c}$ to range from 0.2 to 4.7 , indicating that it is not caused by the dressed interaction. Instead, it is due to the combined effect of radiation pressure forces [60] and the spatial inhomogeneity of the ac Stark shift (see the Supplemental Material [40]).

The results shown in Fig. 4 are encouraging. The achievable values for the lifetime and $\bar{V}_{c}$ are limited by the nonuniform intensity of the dressing beam, rather than by Rydberg excitation. A straightforward way to overcome these effects is to add beam shaping optics to produce a uniform intensity profile. With this improvement, the data in Fig. 4 suggest that the necessary conditions can be maintained for $\sim 3 \mathrm{~ms}$. So far we have not observed excess loss due to Rydberg impurities [31,32,34], perhaps because the spontaneous decay of $|\tilde{e}\rangle$ naturally realizes the stroboscopic scheme proposed to overcome the loss in groundstate dressing experiments [31,34,61].

Last, we discuss possible signatures of Rydberg-dressed interactions. Previous work has considered the motional effects in the fully coherent regime [62-64] or, alternatively, dissipation in the frozen gas regime where motion is neglected $[13,65,66]$. However, the Rydberg-dressed MOT operates in a complex regime where the interaction strength, dissipation, and kinetic energy are all comparable in scale, limiting the usefulness of common approximations for such many-body systems. A possible solution is to combine classical equations of motion with a quantum jump approach for the internal states [20,67], although for the many-body case $N_{c}>1$ this appears to be demanding.

Instead, we considered a mean-field approximation (see the Supplemental Material [40]). We account for the manybody dressed interaction using a step-function approximation $[17,68]$, resulting in an additional detuning that depends on the local density of the cloud. By adding this to our Monte Carlo model, we recursively solve for the density distribution in the presence of interactions and onebody loss from the Rydberg state. The results are shown in Fig. 5 for a uniform intensity $300 \times 100 \mu \mathrm{m}$ dressing beam with power $1 \mathrm{~W}$, for Rydberg fractions $\epsilon^{2}=0.007$ as in Fig. 4, and for $\epsilon^{2}=0.014$. The interaction causes a densitydependent shift in the vertical position of the cloud, associated with a "bending" since the effect is larger in the dense central region than in the wings. These changes in the cloud shape are comparable to those used to study strong interactions in superfluid systems $[69,70]$. Since $\bar{V}_{c}>1$, beyond mean-field effects are also expected. The interaction leads to strong correlations in the scattering of neighboring atoms, which are mapped to and from the motional state of the atoms by the atomic recoil shift, which exceeds the linewidth $\Gamma$. These correlations may lead to novel spatial and dynamical effects not captured by our model.
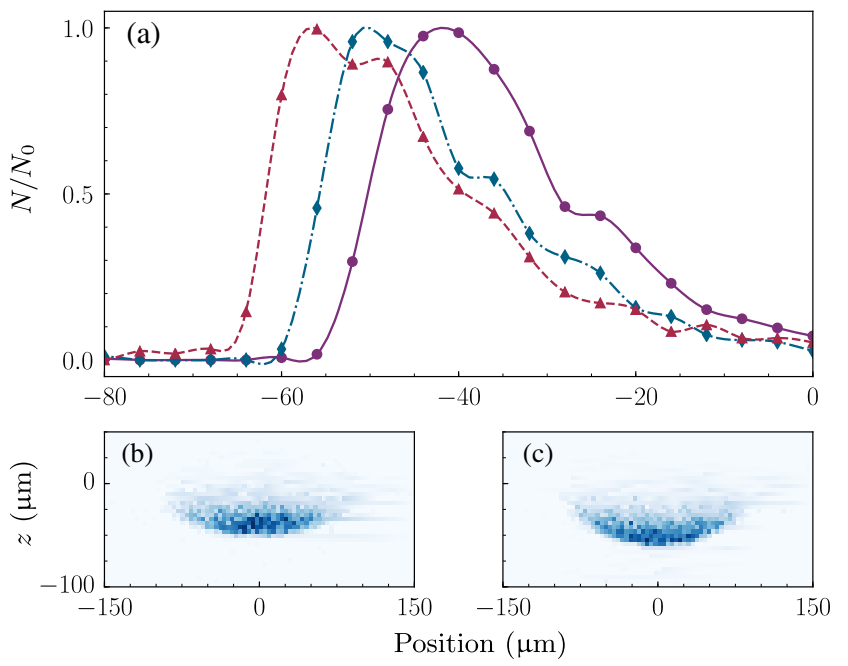

FIG. 5. (a) Vertical slices through the center $(x=0)$ of the simulated density distribution for parameters: noninteracting $\left[\bar{V}_{c}=0\right.$ (the purple circles) $], \epsilon^{2}=0.007, \bar{V}_{c}=2.3$ (the blue diamonds), and $\epsilon^{2}=0.014, \bar{V}_{c}=3.4$ (the red triangles). The lines are guides for the eye. Images show the full 2D density distribution for the (b) noninteracting $\left(\bar{V}_{c}=0\right)$ and (c) interacting $\left(\bar{V}_{c}=3.4\right)$ clouds. 
In summary, we have demonstrated a Rydberg-dressed magneto-optical trap operating with a lifetime in excess of $1 \mathrm{~ms}$ and active laser cooling to sub-microkelvin temperatures. The admixed Rydberg fraction enabled the creation of a hybrid magneto-electro-optical trap that exploits both the large static polarizability of the Rydberg state and the long lifetime afforded by Rydberg dressing. The achievable interaction strength is currently limited by the nonuniform spatial profile of the dressing beam, which is straightforward to overcome in future experiments. A simple model predicts that the Rydberg-dressed interaction should lead to observable changes to the cloud shape. As such, this work represents a promising step towards combining laser cooling with tunable long-range interactions.

The data presented in this paper are available for download [71].

Financial support was provided by EPSRC Grant No. EP/J007021. This project has also received funding from the European Union's Seventh Framework (Grant Agreement No. 612862-HAIRS) and Horizon 2020 (Grant Agreements No. 660028-EXTRYG and No. 640378RYSQ) research and innovation programs.

A. D. B. and N. C. J. contributed equally to this work.

*m.p.a.jones@durham.ac.uk

[1] M. D. Lukin, M. Fleischhauer, R. Cote, L. M. Duan, D. Jaksch, J. I. Cirac, and P. Zoller, Phys. Rev. Lett. 87, 037901 (2001).

[2] H. Labuhn, D. Barredo, S. Ravets, S. de Léséleuc, T. Macrì, T. Lahaye, and A. Browaeys, Nature (London) 534, 667 (2016).

[3] J. Zeiher, R. van Bijnen, P. Schauß, S. Hild, J.-Y. Choi, T. Pohl, I. Bloch, and C. Gross, Nat. Phys. 12, 1095 (2016).

[4] H. Bernien, S. Schwartz, A. Keesling, H. Levine, A. Omran, H. Pichler, S. Choi, A. S. Zibrov, M. Endres, M. Greiner, V. Vuletić, and M. D. Lukin, Nature (London) 551, 579 (2017).

[5] L. Isenhower, E. Urban, X. L. Zhang, A. T. Gill, T. Henage, T. A. Johnson, T. G. Walker, and M. Saffman, Phys. Rev. Lett. 104, 010503 (2010).

[6] M. Saffman, T. G. Walker, and K. Mølmer, Rev. Mod. Phys. 82, 2313 (2010).

[7] T. Wilk, A. Gaëtan, C. Evellin, J. Wolters, Y. Miroshnychenko, P. Grangier, and A. Browaeys, Phys. Rev. Lett. 104, 010502 (2010).

[8] O. Firstenberg, C. Adams, and S. Hofferberth, J. Phys. B 49, 152003 (2016).

[9] J. D. Pritchard, D. Maxwell, A. Gauguet, K. J. Weatherill, M. P. A. Jones, and C. S. Adams, Phys. Rev. Lett. 105, 193603 (2010).

[10] Y. O. Dudin and A. Kuzmich, Science 336, 887 (2012).

[11] T. Peyronel, O. Firstenberg, Q.-Y. Liang, S. Hofferberth, A. V. Gorshkov, T. Pohl, M. D. Lukin, and V. Vuletić, Nature (London) 488, 57 (2012).

[12] D. Maxwell, D. J. Szwer, D. Paredes-Barato, H. Busche, J. D. Pritchard, A. Gauguet, K. J. Weatherill, M. P. A. Jones, and C. S. Adams, Phys. Rev. Lett. 110, 103001 (2013).
[13] H. Weimer, M. Müller, I. Lesanovsky, P. Zoller, and H. P. Büchler, Nat. Phys. 6, 382 (2010).

[14] T. Keating, K. Goyal, Y.-Y. Jau, G. W. Biedermann, A. J. Landahl, and I. H. Deutsch, Phys. Rev. A 87, 052314 (2013).

[15] L. I. R. Gil, R. Mukherjee, E. M. Bridge, M. P. A. Jones, and T. Pohl, Phys. Rev. Lett. 112, 103601 (2014).

[16] L. Santos, G. V. Shlyapnikov, P. Zoller, and M. Lewenstein, Phys. Rev. Lett. 85, 1791 (2000).

[17] I. Bouchoule and K. Mølmer, Phys. Rev. A 65, 041803 (2002).

[18] J. E. Johnson and S. L. Rolston, Phys. Rev. A 82, 033412 (2010).

[19] J. Honer, H. Weimer, T. Pfau, and H. P. Büchler, Phys. Rev. Lett. 105, 160404 (2010).

[20] A. W. Glaetzle, R. Nath, B. Zhao, G. Pupillo, and P. Zoller, Phys. Rev. A 86, 043403 (2012).

[21] N. Henkel, R. Nath, and T. Pohl, Phys. Rev. Lett. 104, 195302 (2010).

[22] G. Pupillo, A. Micheli, M. Boninsegni, I. Lesanovsky, and P. Zoller, Phys. Rev. Lett. 104, 223002 (2010).

[23] F. Cinti, P. Jain, M. Boninsegni, A. Micheli, P. Zoller, and G. Pupillo, Phys. Rev. Lett. 105, 135301 (2010).

[24] M. Boninsegni and N. V. Prokof'ev, Rev. Mod. Phys. 84, 759 (2012).

[25] T. E. Lee, S. Gopalakrishnan, and M. D. Lukin, Phys. Rev. Lett. 110, 257204 (2013).

[26] A. W. Glaetzle, M. Dalmonte, R. Nath, I. Rousochatzakis, R. Moessner, and P. Zoller, Phys. Rev. X 4, 041037 (2014).

[27] A. W. Glaetzle, M. Dalmonte, R. Nath, C. Gross, I. Bloch, and P. Zoller, Phys. Rev. Lett. 114, 173002 (2015).

[28] R. M. W. van Bijnen and T. Pohl, Phys. Rev. Lett. 114, 243002 (2015).

[29] Y.-Y. Jau, A. M. Hankin, T. Keating, I. H. Deutsch, and G. W. Biedermann, Nat. Phys. 12, 71 (2016).

[30] J. Zeiher, J.-y. Choi, A. Rubio-Abadal, T. Pohl, R. van Bijnen, I. Bloch, and C. Gross, Phys. Rev. X 7, 041063 (2017).

[31] J. A. Aman, B. J. DeSalvo, F. B. Dunning, T. C. Killian, S. Yoshida, and J. Burgdörfer, Phys. Rev. A 93, 043425 (2016).

[32] E. A. Goldschmidt, T. Boulier, R. C. Brown, S. B. Koller, J. T. Young, A. V. Gorshkov, S. L. Rolston, and J. V. Porto, Phys. Rev. Lett. 116, 113001 (2016).

[33] S. Helmrich, A. Arias, and S. Whitlock, arXiv:1605.08609.

[34] T. Boulier, E. Magnan, C. Bracamontes, J. Maslek, E. A. Goldschmidt, J. T. Young, A. V. Gorshkov, S. L. Rolston, and J. V. Porto, Phys. Rev. A 96, 053409 (2017).

[35] J. Guo and E. Arimondo, Phys. Rev. A 53, R1224 (1996).

[36] E. Korsunsky and Y. Rozhdestvensky, Phys. Rev. A 52, 3027 (1995).

[37] U. Vogl and M. Weitz, Nature (London) 461, 70 (2009).

[38] B. Zhao, A. W. Glaetzle, G. Pupillo, and P. Zoller, Phys. Rev. Lett. 108, 193007 (2012).

[39] T. Amthor, C. Giese, C. S. Hofmann, and M. Weidemüller, Phys. Rev. Lett. 104, 013001 (2010).

[40] See Supplemental Material at http://link.aps.org/ supplemental/10.1103/PhysRevLett.120.183401, which includes Refs. [41-48], for more detailed discussions.

[41] K. R. Overstreet, P. Zabawa, J. Tallant, A. Schwettmann, and J. P. Shaffer, Opt. Express 13, 9672 (2005). 
[42] C. G. Townsend, N. H. Edwards, C. J. Cooper, K. P. Zetie, C. J. Foot, A. M. Steane, P. Szriftgiser, H. Perrin, and J. Dalibard, Phys. Rev. A 52, 1423 (1995).

[43] M. Drewsen, P. Laurent, A. Nadir, G. Santarelli, A. Clairon, Y. Castin, D. Grison, and C. Salomon, Appl. Phys. B 59, 283 (1994).

[44] S. Grego, M. Colla, A. Fioretti, J. H. Müller, P. Verkerk, and E. Arimondo, Opt. Commun. 132, 519 (1996).

[45] C. Gabbanini, A. Evangelista, S. Gozzini, A. Lucchesini, A. Fioretti, J. H. Müller, M. Colla, and E. Arimondo, Europhys. Lett. 37, 251 (1997).

[46] A. Vorozcovs, M. Weel, S. Beattie, S. Cauchi, and A. Kumarakrishnan, J. Opt. Soc. Am. B 22, 943 (2005).

[47] H. Katori, T. Ido, Y. Isoya, and M. Kuwata-Gonokami, Phys. Rev. Lett. 82, 1116 (1999).

[48] A. M. Steane, M. Chowdhury, and C. J. Foot, J. Opt. Soc. Am. B 9, 2142 (1992).

[49] D. Boddy, Ph.D. thesis, Durham University, 2014.

[50] J. Millen, Ph.D. thesis, Durham University, 2011.

[51] T. H. Loftus, T. Ido, M. M. Boyd, A. D. Ludlow, and J. Ye, Phys. Rev. A 70, 063413 (2004).

[52] C. L. Vaillant, M. P. A. Jones, and R. M. Potvliege, J. Phys. B 45, 135004 (2012).

[53] E. M. Bridge, N. C. Keegan, A. D. Bounds, D. Boddy, D. P. Sadler, and M. P. A. Jones, Opt. Express 24, 2281 (2016).

[54] R. K. Hanley, P. Huillery, N. C. Keegan, A. D. Bounds, D. Boddy, R. Faoro, and M. P. A. Jones, J. Mod. Opt. 65, 667 (2018).

[55] T. Middelmann, S. Falke, C. Lisdat, and U. Sterr, Phys. Rev. Lett. 109, 263004 (2012).
[56] J. A. Sherman, N. D. Lemke, N. Hinkley, M. Pizzocaro, R. W. Fox, A. D. Ludlow, and C. W. Oates, Phys. Rev. Lett. 108, 153002 (2012).

[57] A. Bounds, Ph.D. thesis, Durham University, 2017 (unpublished).

[58] W. Bowden, R. Hobson, P. Huillery, P. Gill, M. P. A. Jones, and I. R. Hill, Phys. Rev. A 96, 023419 (2017).

[59] The $n=37$ state is very close to a Förster resonance leading to a large (and rather uncertain) $C_{6}$ value.

[60] D. W. Sesko, T. G. Walker, and C. E. Wieman, J. Opt. Soc. Am. B 8, 946 (1991).

[61] C. Gaul, B. J. DeSalvo, J. A. Aman, F. B. Dunning, T. C. Killian, and T. Pohl, Phys. Rev. Lett. 116, 243001 (2016).

[62] T. Macrı and T. Pohl, Phys. Rev. A 89, 011402 (2014).

[63] M. Genkin, S. Wüster, S. Möbius, A. Eisfeld, and J. M. Rost, J. Phys. B 47, 095003 (2014).

[64] L. F. Buchmann, K. Mølmer, and D. Petrosyan, Phys. Rev. A 95, 013403 (2017).

[65] N. Malossi, M. M. Valado, S. Scotto, P. Huillery, P. Pillet, D. Ciampini, E. Arimondo, and O. Morsch, Phys. Rev. Lett. 113, 023006 (2014).

[66] M. Marcuzzi, E. Levi, S. Diehl, J. P. Garrahan, and I. Lesanovsky, Phys. Rev. Lett. 113, 210401 (2014).

[67] N. Šibalić, C. G. Wade, C. S. Adams, K. J. Weatherill, and T. Pohl, Phys. Rev. A 94, 011401 (2016).

[68] J. B. Balewski, A. T. Krupp, A. Gaj, S. Hofferberth, R. Löw, and T. Pfau, New J. Phys. 16, 063012 (2014).

[69] S. L. Cornish, N. R. Claussen, J. L. Roberts, E. A. Cornell, and C. E. Wieman, Phys. Rev. Lett. 85, 1795 (2000).

[70] T. Lahaye, T. Koch, M. Fattori, J. Mettz, A. Griesmaier, S. Giovanazzi, and T. Pfau, Nature (London) 448, 672 (2007).

[71] DOI: 10.15128/r1rv042t056. 